\title{
L'Homme
}

Revue française d'anthropologie

$190 \mid 2009$

Varia

\section{Valérie Lécrivain, ed., Clientèle guerrière, clientèle foncière et clientèle électorale}

Gautier Bourdeaux

\section{(2) OpenEdition}

1 Journals

Édition électronique

URL : http://journals.openedition.org/lhomme/28713

DOI : 10.4000//homme.28713

ISSN : 1953-8103

Éditeur

Éditions de l'EHESS

Édition imprimée

Date de publication : 1 janvier 2009

Pagination : 206-207

ISSN : 0439-4216

\section{Référence électronique}

Gautier Bourdeaux, "Valérie Lécrivain, ed., Clientèle guerrière, clientèle foncière et clientèle électorale »,

L'Homme [En ligne], 190 | 2009, mis en ligne le 03 janvier 2017, consulté le 24 septembre 2020. URL :

http://journals.openedition.org/lhomme/28713 ; DOl : https://doi.org/10.4000//homme.28713

Ce document a été généré automatiquement le 24 septembre 2020.

(c) École des hautes études en sciences sociales 


\title{
Valérie Lécrivain, ed., Clientèle guerrière, clientèle foncière et clientèle électorale
}

\author{
Gautier Bourdeaux
}

\section{RÉFÉRENCE}

Valérie LÉCRIVAIN, ed., Clientèle guerrière, clientèle foncière et clientèle électorale. Histoire et anthropologie, Dijon, Éd. universitaires de Dijon, 2007, 250 p., notes bibliogr., index, ill.

1 LA CLIENTÈLE et le clientélisme sont des phénomènes qui ont suscité nombre d'études émanant des divers spécialistes des sciences sociales et des sciences politiques. Pourtant, malgré ces travaux essentiels, le concept même de clientèle, envisagé en tant que rapport social spécifique, et dépassant une société donnée ou une époque déterminée, méritait une analyse approfondie. C'est la raison pour laquelle s'est tenu, durant les années 2003 à 2005, sous l'égide du Laboratoire d'anthropologie sociale, et sous la direction de Valérie Lécrivain, un séminaire sur la clientèle qui s'est conclu par un colloque de deux journées. L'ouvrage recensé ici parachève ces travaux.

2 Son titre éclaire le lecteur quant à la méthode retenue par les auteurs : confronter les expertises des anthropologues, des historiens et des archéologues sur le thème de la clientèle. Une lecture rapide dudit titre faisant référence à une clientèle guerrière, foncière ou électorale, ne doit cependant pas induire en erreur et laisser accroire que la notion ne présenterait pas une unité fondamentale singulière. Bien au contraire, l'intérêt ici est de montrer, à partir des analyses des diverses disciplines, que la clientèle est un phénomène existant dans de nombreuses sociétés et/ou périodes historiques.

3 C'est ainsi qu'Élisabeth Deniaux aborde ce phénomène à l'époque de la république romaine (pp. 161-172). Le rôle essentiel et caractéristique de la relation client/patron y fut en effet tel que c'est bien cette société qui fournit généralement le modèle du 
concept de clientèle et qui se trouve au départ des analyses sur ce thème. La présence de relations de clientèle est toutefois probable dans l'Antiquité au-delà de la civilisation romaine. C'est ce que montre l'article d'Alain Testart et Luc Baray, relatif aux ambactes et soldures en Gaule, «figures du compagnonnage guerrier » (pp. 51-84). C'est encore ce qu'envisage Christophe Pébarthe dans son article sur la Grèce ancienne au siècle de Périclès (pp. 173-197). Le monde méditerranéen postérieur à la civilisation antique a, lui aussi, suscité nombre d'études quant au phénomène clientélaire comme le rappelle la contribution de Gérard Delille qui aborde plus spécialement le contrôle des circuits financiers en Italie du Sud (pp.33-50). L'existence d'une telle relation est, en outre, démontrée en France aux XVI ${ }^{\mathrm{e}}$ et XVII ${ }^{\mathrm{e}}$ siècles. En ce sens, Ariane Boltanski examine les relations des Nevers avec leur clientèle au cours de la seconde moitié du XvII ${ }^{\mathrm{e}}$ siècle (pp.115-135), tandis que Jean Duma souligne la difficulté pour l'historien de saisir ce type de relation sociale à l'époque moderne (pp.99-113). Le clientélisme dépasse cependant le seul continent européen ou le bassin méditerranéen. Henri Médard démontre ainsi qu'un phénomène comparable se retrouve dans l'Afrique des grands lacs et, plus précisément, au royaume du Buganda durant la période 1750-1920 (pp. 85-98). Colette Callier, quant à elle, consacre une étude au pouvoir local dans un municipe de l'Agreste du Pernambouc au Brésil (pp.199-218). Enfin, une illustration mélanésienne de rapports de clientèle est fournie avec les milices privées des big men ou des hauts gradés de certaines sociétés, notamment du Vanuatu et d'Irian Jaya, relations décrites par Valérie Lécrivain (pp. 136-160).

L'ouvrage ne se contente pas d'un brillant panorama du fait clientélaire, son ambition est non seulement de montrer que les relations de clientèle sont présentes dans diverses sociétés mais également de contribuer à éclairer ce concept et d'en préciser le sens anthropologique. Cela explique la structure de cette publication, dans laquelle les articles, consacrés à des sociétés et des époques spécifiques, sont précédés d'une introduction définissant la clientèle par comparaison avec les concepts proches (pp.13-31), et se concluent par un article d'Alain Testart proposant une définition argumentée de cette notion (pp. 219-239).

Un des apports essentiels de l'ouvrage tient d'ailleurs à la volonté des auteurs d'opérer une distinction entre la clientèle, qui seule relève de leur analyse, et le phénomène du clientélisme. La clientèle traduit, en effet, l'existence d'une structure sociale spécifique, tandis que le clientélisme, est une notion, utilisée notamment par les politologues, qui vise à décrire le fonctionnement ou le dysfonctionnement d'une société. Un critère complémentaire de distinction est également avancé : dans le clientélisme politique, patrons et clients appartiennent à un même groupe, ethnie ou parti, alors que la clientèle correspond à un lien personnel entre un patron et un individu ou un groupe d'individus. Par conséquent, même si clientèle et clientélisme sont souvent concomitants, il peut exister des sociétés caractérisées par la présence de liens de clientèle sans clientélisme et inversement.

6 Au-delà de cette distinction essentielle, les auteurs de l'ouvrage entendent rappeler la différence entre clientèle et vassalité, située au niveau de la formalisation ou non des relations en cause. Alors que cette formalisation est présente dans la vassalité - par un serment ou par un contrat solennel -, les rapports de clientèle restent dans le domaine de l'informel, ce qui n'interdit d'ailleurs pas, comme le démontre l'exemple romain, le droit d'appréhender cette relation sociale. 
7 La méthode comparative utilisée dans cet ouvrage et la délimitation de la notion opérée suscitent alors quelques réflexions. Ainsi ne pourrait-on pas esquisser certaines subdivisions ? Une première typologie, directement suggérée dès le titre, montre que la clientèle est susceptible de correspondre à diverses fonctions (guerrière, électorale et foncière). En outre, une seconde typologie apparaît lorsque l'on distingue, d'une part, un patronage dyadique qui constitue probablement le schéma de base du rapport de clientèle, et, d'autre part, un patronage communautaire entre un patron et un groupe d'individus envisagé, comme le suggère Alain Testart, comme personne morale (pp. 236 sq.). Le caractère personnel du rapport social ne saurait être exactement de même intensité dans ces deux cas : il existerait, en quelque sorte, une clientèle et une quasiclientèle. Ces quelques réflexions montrent la richesse et l'intérêt de l'ouvrage publié sous la direction de Valérie Lécrivain : il permet non seulement de faire une synthèse pluridisciplinaire de la recherche relative à la clientèle mais aussi, au-delà, il entend montrer en quoi la clientèle est un type de rapport social spécifique. Ce travail ouvre, à cet égard, de stimulantes perspectives.

\section{AUTEURS}

\section{GAUTIER BOURDEAUX}

Université Paris-V Descartes, Faculté de Droit, Paris.

gautier.bourdeaux@univ-paris5.fr 